\title{
Relative Expression of Soluble tnf Versus Transmembrane tnf in Spondyloarthritis
}

\author{
Huriatul Masdar
}

\begin{abstract}
TNF is important in rheumatoid arthritis (RA) and spondyloarthritis ( $\mathrm{SpA}$ ) pathogenesis. Recently reports showed sTNF levels were significantly lower in SpA than RA synovial fluid (SF). Therefore, the differential regulation of TNF in both diseases were investigated focusing on the balance of sTNF-tmTNF and both TNF receptors expression. Synovial fluid from $22 \mathrm{RA}$ and $25 \mathrm{SpA}$ were analyzed by ELISA. sTNF was confirmed significantly higher in RA than SpA. Total TNF of synovial tissues assessed by qPCR showed similar levels, suggesting a higher tmTNF/sTNF ratio in SpA. The levels of sTNF-R1 and sTNF-R2 were significantly lower in SpA than RA SF. Interestingly, sTNF-R2/sTNF-R1 increased in SpA. qPCR on synovial tissue showed similar mRNA levels for TNF-R2 but decrease of TNF-R1 in SpA. IL-6R levels did not showed differences and TIMP-3 tended to be higher in RA than in SpA SF. In conclusion, we observed a shift from sTNF/TNF-R1 in RA to tmTNF/TNF-R2 in SpA.
\end{abstract}

Keywords: spondyloarthritis - synovial fluid - TNF - TNFRs - TACE

TNF is a pleiotropic molecule produced predominantly by activated macrophages and $\mathrm{T}$ cells, but it can also be produced by other cell types such as mast cells, natural killer (NK) cells, neutrophils, endothelial cells, smooth and cardiac muscle cells, fibroblast and osteoclast. It has been well known that this molecule has beneficial activities in immune regulation and host defense mechanism as well as hazardous effects as proinflammatory and cytotoxic cytokine. ${ }^{1,2}$

There are two forms of TNF: transmembrane TNF (tmTNF) and soluble TNF (sTNF). tmTNF, also known as pro-TNF, is a $26 \mathrm{kDa}$ protein which is expressed on the cell plasma membrane. Its extracellular domain can be cleaved into soluble form of TNF, a $17 \mathrm{kDa}$ molecule, by the matrix metalloproteinase known as TNF alfa converting enzyme (TACE) or ADAM 17. Both forms of TNF are active in their trimeric forms but their biological activities are partially differen. ${ }^{3,4}$

\footnotetext{
1 Corresponding author: Department of Histology Faculty of Medicine Riau University Pekanbaru Indonesia dr_huriatul@yahoo.co.id
}

The functions of TNF are mediated via two different cell surface receptors: TNF-R1 (p55TNFR or CD 120a) and TNF-R2 (p75TNFR or CD 120b). Both receptors have the same extracellular domain containing cystein-rich sub-domains but the intracellular domain of TNF-R1 contains a death domain which is not expressed in TNF-R2. ${ }^{5}$ Similarly to tmTNF itself, both TNF receptors can be cleaved by TACE into soluble TNF-Rs that can neutralize the TNF activity. ${ }^{6,7}$ Whereas tmTNF binds both receptors, sTNF preferentially binds to TNF$\mathrm{R} 1$. The differences in the intracellular domain as well as the preferential binding of sTNF to TNFR1 may lead to different biological effects of both receptors as demonstrated by different experimental models. However, it is as yet unclear in how far these differences contribute to human pathology. ${ }^{8-}$ 10

As indicated, TACE (ADAM17, CD156b or EC 3.4.24) is the enzyme responsible for limited proteolysis of tmTNF at Ala76 and Val77, leading to the shedding of sTNF. TACE is a type I transmembrane protein which belongs to a disintegrin and metalloproteinase domain (ADAM) family, 
which in turn belongs to metzincin super-family. This enzyme is synthesized as zymogen. It has a pro-domain which is considered to act as proteinase activity inhibitor, a catalytic domain, a disintegrin and cystein-rich region, a trans-membrane segment and a cytoplasmic tail. The presence of free cystein residue in the pro-domain with zinc in the active site of TACE maintains its activity. 4,12,13

Transmembrane-TNF is not the only substrate of TACE. Indeed, TACE"Zn"'Zn mice display a defect which resembles TGF alpha deficiency. Accordingly, the release of TGF alpha from the cell membrane was inhibited in TACE"Zn" $" \mathrm{Zn}$ mice, proving the pivotal role of TACE in TGF-á shedding. ${ }^{14}$ Similarly, TACE is responsible for the shedding of L-selectin, Amyloid Precursor Protein (APP), and IL-1R. ${ }^{15}$ The shedding of IL-6R in osteoblast like cells was also reported to be partially mediated by TACE. ${ }^{16}$

The regulation of TACE activity is not well understood. The rate of TACE activity to shed transmembrane proteins increased within minutes in the presence of additional cell activators such as phorbol ester. The enhancement of TACE shedding rate could be blocked by inhibiting Mitogen Activated Protein Kinase (MAPK) cascade but the exact mechanism of this blocking remains poorly understood. ${ }^{17}$ Interestingly, Tissue Inhibitor of Metalloproteinase-3 (TIMP-3) was reported to specifically inhibit TACE activity. However, whether this protein functions as physiological regulator of this enzyme is also not known yet. ${ }^{18}$

Rheumatoid arthritis (RA) and spondyloarthritis $(\mathrm{SpA})$ are the two most prevalent forms of chronic inflammatory peripheral joint diseases. Macrophages and their pro-inflammatory products play a crucial role in both pathologies as evidenced by the correlation of macrophage numbers with disease severity at the site of inflammation ${ }^{8-10}$ and the clinical and histological response to TNF blockade. ${ }^{19,20}$ Recent researches on macrophages in synovitis have indicated that the synovial tissue is infiltrated by different macrophage subsets in SpA versus RA. ${ }^{21-22}$ Indeed, macrophages are a heterogeneous population of cells with distinct phenotypic and functional subsets depending on the way they are activated. ${ }^{8,23}$ We proposed that RA immunopathology is driven by classically activated macrophages (CAM), the main source of soluble pro-inflammatory cytokines such as IL-1â and TNF, whereas alternatively activated macrophages (AAM) predominate in SpA. In agreement with this concept, it was recently reported that synovial fluid levels of sTNF are significantly lower in SpA than in RA despite similar degrees of joint inflammation. ${ }^{24}$

The apparent discrepancy between the local levels of sTNF, which are lower in SpA than in RA, and the response to TNF blockade, which is often better in SpA than in RA, raises the question whether tmTNF rather than STNF may be an important driver of synovial inflammation in SpA. We thus propose the hypothesis that the ratio of $\mathrm{sTNF} / \mathrm{tmTNF}$ is lower in SpA versus RA synovitis, with a selective increase of tmTNF in SpA. This disbalance between soluble and trans-membrane TNF could be either by an intrinsic feature of $\mathrm{SpA}$, a consequence of reduced TACE activity, or the results of the specific differences in macrophage subsets in both diseases in which AAM, which predominate in SpA, may express higher levels of tmTNF than CAM but secrete lower levels of sTNF. The proposed disbalance could also be paralleled by altered TNFR expression as these receptors are also shed by TACE. This would further influence the functional impact of the disbalance.

\section{METHODS}

\section{Samples}

Synovial fluids and frozen synovial tissues were obtained from Amsterdam Medical Centre (AMC) BioBank collections. These samples were obtained from patients in AMC who are diagnosed as RA and SpA patients that fulfill the criteria of American College of Rheumatology (ACR) and European Spondyloarthropathy Study Group (ESSG), respectively. Ethical clearance was approved by Ethical Comittee of Amsterdam Medical Centre. Blood samples were obtained from healthy donors who work at Experimental Immunology Department of AMC.

\section{Soluble TNF ELISA}

Sandwich ELISA was performed to determine the level of sTNF in SF and supernatants of 
polarized macrophages by using antibody pair purchased from Sanquin, Netherlands. The maxisorb 96 wells flat bottom plate (Nunc, Denmark) were coated with 1:100 of captured antibody overnight, at $4^{\circ} \mathrm{C}$ and blocked with fat-milk diluted in PBS (1:50). Undiluted samples were applied, followed by incubation with biotinylated detection antibody (1:100). The detection antibody was probed with strepatavidine-HRP (1:10000) and colorized with substrate solution containing hydrogen peroxidase and tetramethylbenzidine (TMB). The optical density (OD) of reaction was read by using ELISA reader (Model 680 Microplate Reader, Bio-Rad) at $450 \mathrm{~nm}$ and analyzed with Microplate Manager 5.2 programme.

\section{Solubel TNF-R1, sTNF-R2 and IL-6R ELISA}

The levels of sTNF-R1 (Bender Medsystems, $\mathrm{GmbH}$ ), sTNF-R2 (Bender Medsystems, GmbH) and Il-6R (R\&D, Abingdon, UK) in synovial fluid were determined by using ELISA commercial kits and following manufacturer's protocol. Ten times dilution of samples were applied in each ELISA.

\section{TIMP-3 ELISA}

Duo set antibody for ELISA (R\&D, Abingdon, UK) was used to detect the level of TIMP-3. The maxisorb 96 wells plate (Nunc, Denmark) was coated with $2 \mathrm{ug} / \mathrm{ml}$ of captured antibody in PBS for overnight at room temperature. PBS containing $1 \%$ BSA was used to block an unspecific binding. Five times dilution of samples were applied, followed by incubation with detection antibody (2 $\mathrm{ug} / \mathrm{ml})$. Streptavidine-HRP (1:10000, Sanquin) was used to probe the antibody followed by colorization with substrate solution containing hydrogen peroxidase and tetramethylbenzidine (TMB).

\section{Monocytes Isolation and In Vitro Polarization}

Monocytes were isolated from peripheral blood of healthy donor by using Ficoll gradient centrifugation and plastic adhesion. The isolated monocytes were cultured in IMDM 10\% FCS and polarized into macrophages subtypes M1a, M2a and M2c by stimulating the cells with IFNã $(50 \mathrm{ng} / \mathrm{ml}$, R\&D Systems), IL-4 (40 ng/ml, R\&D Systems) and
IL-10 (50 ng/ml, R\&D Systems) for 3 days, respectively. One day additional stimulation with LPS ( $1 \mathrm{ug} / \mathrm{ml}$, Sigma Aldrich) was performed in specific experiments.

\section{Quantitative Polymerase Chain Reaction (qPCR)}

qPCR was used to measure total TNF, TNF-R1 and TNF-R2 in synovial tissue biopsies from a cohort study to early and untreated 10 RA and 10 SpA patients. mRNA from synovial tissues were extracted by using RNA STAT-60 kit (Tel-Test, Inc) following manufacturer's protocol. RevertAid $\mathrm{H}$ Minus First Strand cDNA Synthesis kit (Fermentas Lfe Sciences) was used to convert mRNA into cDNA. The genes of interest were amplified and quantified by using Sybr@Green qPCR from Applied Biosystems and the results were analysed by Step One Software v.2.1 from the same company. The primer sequences used were CCCATGTTGTAGCAAACCCT (forward) and TGAGGTACAGGCCCTCTGAT (reverse) for TNF, TACCGGCATTATTGGAGTGA (forward) and GCACACGGTGTTCTGTTTCT (reverse) for TNF-R1, and ACACCGTGTGTGACTCCTGT (forward) and AGTTTCCACCTGGTCAGAGC (reverse) for TNF-R2. The samples were applied in duplo and GAPDH was used as the house keeping gene to set up the baseline.

\section{Antibody Titration for Immuno histochemistry Staining}

Mouse monoclonal antibodies against TNF (Thermo Sciences), TNF-R1 (Abcam) and TNF-R2 (Abcam) were used to detect each cytokine in synovial tissues. In these series of pilot experiments, we determined the optimal concentration of the primary antibodies. Frozen synovial tissue were fixed with acetone and blocked with $10 \mathrm{ul} 30 \% \mathrm{H}_{2} \mathrm{O}_{2}$ in $1 \mathrm{ml} 0.1 \%$ Sodium Azide in PBS, followed by incubation with serial dilutions of primary antibodies $(0.5,2$ and $10 \mathrm{ug} / \mathrm{ml})$ against total TNF (Thermo Sciences), TNF-R1 (Abcam) and TNF-R2 (Abcam) for overnight at $4^{\circ} \mathrm{C}$. Serial dilutions of anti mouse $\mathrm{IgG}_{1}$ antibody (Dako) was used as the negative control. Goat anti mouse $\mathrm{IgG}_{1}$ (Dako) in PBS/1\%BSA/10\%Normal Human Serum was used 
as the secondary antibody. Before probing the antibody with Strepatividine-HRP (Dako), BiotinTyramine in amplification buffer $(0.05 \mathrm{M}$ Tris, 1:750) was used to enhance the signal. After staining the sections with AEC solution kit (Vector Laboratories, Inc., Burlingame, CA), the sections were counterstained with Hematoxylin (Fluka, Sigma Aldrich $\mathrm{GmbH}$ ). The staining was analyzed by light microscopy. The stained synovial tissues will be scored qualitatively from $0-3$ by two researchers.

\section{Flow Cytometry}

Macrophages polarized by IFNã, IL-4, IL-10 and subsequently stimulated with LPS were analyzed for expression of the prototypical phenotypic markers CD14 which is highly expressed with LPS stimulation, CD200R which is upregulated by IL-4 stimulation, and CD163 as well as for tmTNF expression. FcR blocking reagent for human cells (MACS, Miltenyi Biotec, $\mathrm{GmbH}$ ) was used to block FcãR ( $1 \mathrm{ug} / 10^{5}$ cells). The cells were incubated with mouse anti-human CD200R-Alexa $488 \mathrm{IgG}_{1}(0.15 \mathrm{ng} / \mathrm{ml}, \mathrm{AbD}$ serotec $)$, mouse antihuman CD163-Phycoerythrine (PE) $\operatorname{IgG}_{1}$ (3:50, BD Biosciences), mouse anti-human CD14-PerCP-Cy $5.5 \mathrm{IgG}_{1}(0.05 \mathrm{ng} / \mathrm{ml}$, eBiosciences $)$ and anti-human TNFá-Allophycocyanin (APC)(3:50, BD Biosciences). For isotype control, mouse IgG1Alexa Flour 488 isotype control $(0.15 \mathrm{ng} / \mathrm{ml}, \mathrm{AbD}$ Serotec), mouse IgG1-PE isotype control ( $0.15 \mathrm{ng} /$ $\mathrm{ml}$, BD Biosciences), mouse IgG1-PerCP-Cy-5.5 isotype control $(0.05 \mathrm{ng} / \mathrm{ml}$, eBiosciences $)$ and mouse IgG1-APC isotype control $(2.5 \mathrm{ng} / \mathrm{ml}, \mathrm{BD}$ Biosciences) were used. To assess tmTNF, we also compared the polarized macrophages with and without cell permeabilization. In permeabilized cells, saponin buffer was used in antibody mixture instead of PBA. The stained cells were detected with FACS Calibur system (BD Biosciences). Flow Jo v.8.8.6 software was used to analyze the results.

\section{Statistic Analysis}

The SF levels of sTNF, sTNFR1, sTNFR2, sTNFR2/sTNFR1 ratio, IL-6R and TIMP-3 between RA versus SpA were analyzed by using the nonparametric Mann Whitney-U test. The data are presented as median with interquartile range. $P$ value less than 0.05 were considered as statistically significant.

\section{RESULTS}

\section{STNF, sTNFR1 and sTNFR2 in Synovial Fluid}

The levels of sTNF, sTNFR1 and sTNFR2 were assessed by ELISA in 47 synovial fluid samples ( 22 RA and $25 \mathrm{SpA}$ ). Confirming our previous results, SF sTNF levels were significantly lower in SpA compared to RA ( $\mathrm{p}=0.0117)$. Similarly, SF sTNF$\mathrm{R} 1(\mathrm{p}<0.0001)$ and sTNF-R2 $(\mathrm{p}=0.0004)$ levels were decreased in SpA versus RA. Interestingly, the ratio of sTNF-R2 to sTNF-R1 was significantly higher in $\mathrm{SpA}$ than RA ( $\mathrm{p}=0.0002)$ (figure 1).

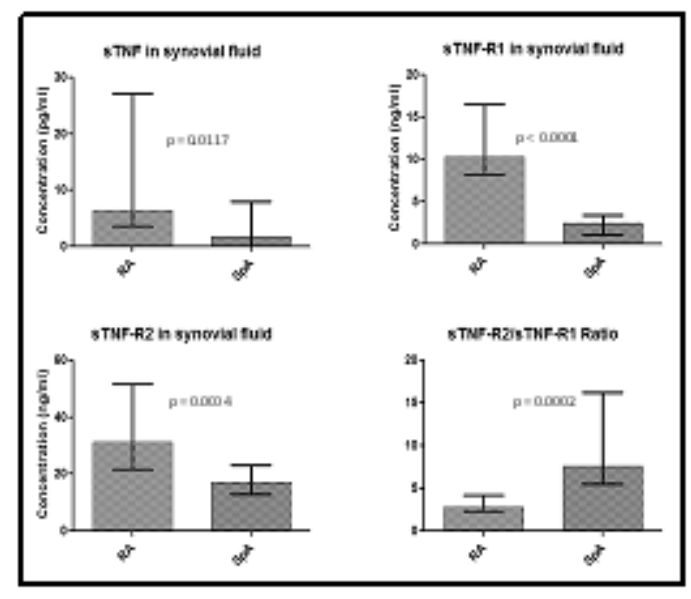

Figure 1. The levels of sTNF, sTNF-R1, sTNF-R2 and sTNF-R2/sTNF-R2 ratio.

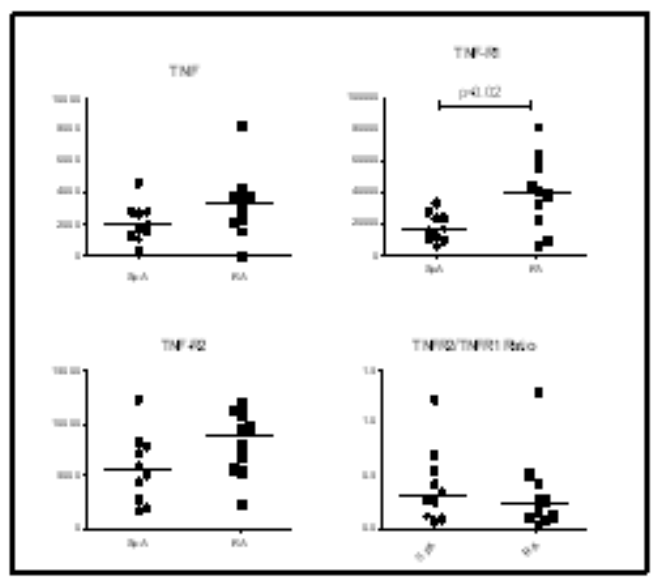

Figure 2. Total TNF, TNF-R1 and TNF-R2 expression in synovial biopsies from a cohort study. 


\section{Total TNF, TNF-R1 and TNF-R2 in Synovial Tissues}

Synovial biopsies from a cohort study to early and untreated of $10 \mathrm{RA}$ and $10 \mathrm{SpA}$ patients were analyzed for their mRNA expression of TNF, TNF$\mathrm{R} 1$ and TNF-R 2 by using qPCR. As shown in figure 2, the TNF mRNA expression from RA was slightly higher than SpA but it was not significantly different $(\mathrm{p}=0.123)$. Similarly, TNF-R2 was also not significantly higher in $\mathrm{RA}$ than $\mathrm{SpA}(\mathrm{p}=0.123)$. The expression of TNF-R1 was significantly higher in RA than SpA ( $p=0.02)$. The ratio of TNF-R1 over TNF-R2 was slightly higher in SpA than in RA but there was no statistically different $(p=0.5705)$.
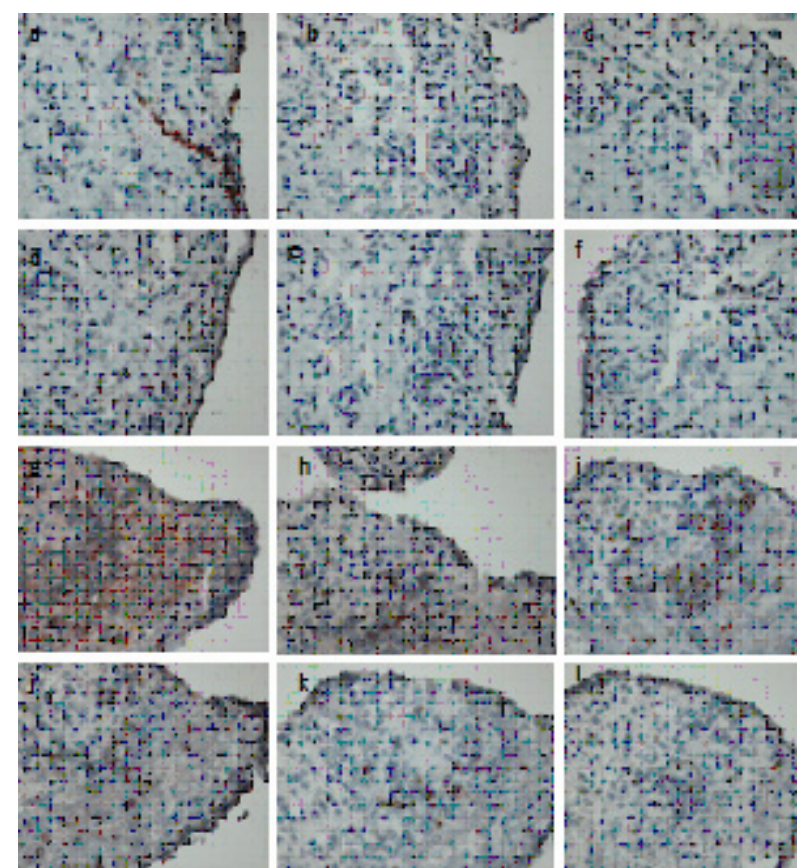

Figure 3.

Optimization of antibodies against TNF, TNF-R1 and TNF-R2 in IHC. The first row $(\mathrm{a}, \mathrm{b}, \mathrm{c})$ is the negative control, the second, the third and the last rows are synovial tissues stained for their TNF, TNF-R1 and TNF-R2 expression, respectively. The column showed the serial dilutions of antibodies used which are $10 \mathrm{ug} / \mathrm{ml}$ (left), 2 $\mathrm{ug} / \mathrm{ml}$ (middle) and $0.5 \mathrm{ug} / \mathrm{ml}$ (right).

To confirm the synovial expression of total TNF, TNF-R1 and TNF-R2 at protein level, we started to perform immunohistochemistry (IHC) experiments on frozen sections of inflamed synovial tissues. As shown in figure 3, we were able to optimize that staining for TNF-R1 and TNF-R2. By comparing to the negative control, TNF-R1 and TNF-R2 were optimal to use in the concentration 2 $\mathrm{ug} / \mathrm{ml}$. However, TNF was expressed very low and almost nothing although in the high antibody concentration $(10 \mathrm{ug} / \mathrm{ml})$. We are now still working on it.

\section{TACE expression}

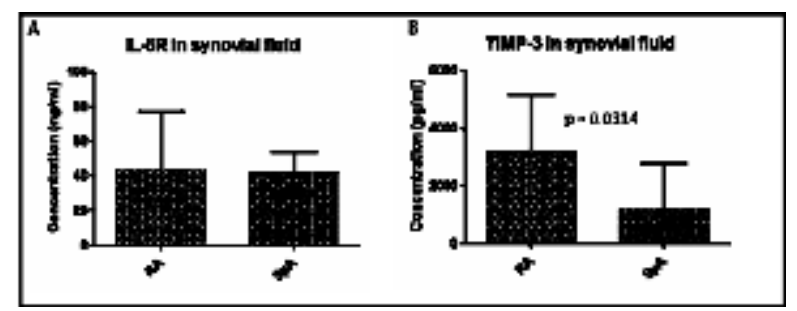

Figure 4. IL-6R and TIMP-3 levels in synovial fluids from RA and SpA patients.

As TACE, the enzyme responsible for cleavage of sTNF as well as sTNF-R1 and sTNF-R2, can not be detected in extracellular fluids, we firstly carried out an ELISA to detect the level of SF IL-6R as this receptor is also cleaved by TACE. Analysis of 20 RA and 20 SpA SF samples showed no differences in sIL-6R ( $p=0.2791)$ (figure 4A).

Accordingly, the TACE inhibitor TIMP-3 was not increased but rather decreased in SpA SF compared to RA SF ( $p=0.0314$ ) (Figure 4B). Both findings suggest that the observed differences in sTNF and sTNF-R1 and s-TNF-R2 are not directly related to TACE expression or activity.

\section{Transmembrane TNF Expression in Polarized Macrophages}

To investigate whether differential mTNF expression is related to the differences in macrophage polarization between both diseases, we assessed the expression of these molecules by flow cytometry on peripheral blood monocytes-derived macrophages from healthy individuals which were polarized in vitro into M1a (CAM), M2a and M2c (AAM) by IFNã, IL- 4 and IL-10 stimulation, respectively. CD14 expression was downregulated by IL-4 and IFNã stimulation. Under IL-10 stimulation, the expression of CD14 was almost the 
same as control. IL-4 stimulation upregulated the expression of CD200R and IL-10 upregulated the expression of CD163 (figure 5), indicating that the cells have been correctly polarized in our experiment. However, we were not able to detect the expression of tmTNF both in absence and presence of additional LPS stimulation. Permeabilization of the cells to assess the transmembrane TNF did not show any differences from non-permeabilized cells (data not shown).

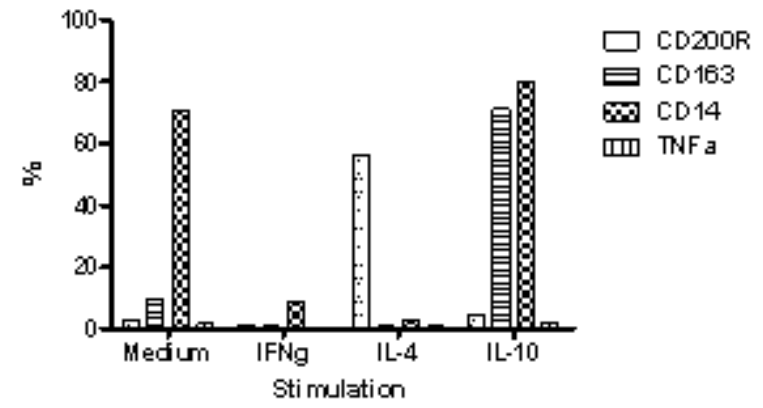

Figure 5. Polarized macrophages with IFNã, IL-4 and IL-10 stimulations.

As internal control, the supernatant of the polarized macrophages was analyzed by ELISA to detect the present of sTNF. LPS failed to induce production and secretion of TNF in IL-10 polarized macrophages. On the contrary, polarization with IFNã or IL-4 almost doubled the LPS-induced TNF production (figure 6).

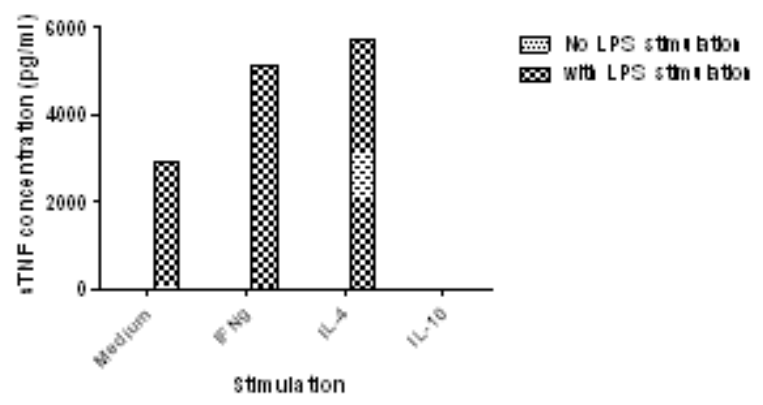

Figure 6. Soluble TNF level in supernatants of macrophages polarized with IFNã, IL-4 and IL-10 in the presence and absence of additional LPS stimulation.

\section{DISCUSSION}

Excellent clinical and biological response of
RA and SpA to TNF blocker treatment proved that TNF plays a pivotal role in pathogenesis of both diseases. The significantly lower SF levels of sTNF in SpA compared to RA led us to the hypothesis that tmTNF, and not sTNF, is a key mediator of SpA synovitis and that this may explain pathological differences between SpA and RA.

We investigated the level of sTNF in synovial fluid from RA and SpA patients by ELISA. The level of sTNF in synovial fluids from RA patients was significantly higher than from SpA. This result confirmed the previous investigations. ${ }^{24}$ The SF levels of sTNF-R1 and sTNF-R2 were also higher in RA than SpA, but interestingly, the ratio sTNFR2/sTNF-R1 was significantly higher in SpA than RA. Together with the similar total TNF but lower TNF-R1 mRNA levels in SpA synovium, this suggests a predominance of tmTNF/TNF-R2 signaling in SpA compared to sTNF/TNF-R1 signaling in RA.

We have started a number of experiments to confirm these ELISA and qPCR data by immunohistochemistry on frozen sections of synovial tissue. To assess the right working concentration of each antibody, we performed a pilot project with serial dilutions of each antibody. We have now set up a protocol for TNF-R1 and TNFR2 staining but were, unfortunately, not able to obtain a reliable TNF immunostaining. Some technical points have been discussed, however, reliable TNF immunostaining was still unable to be performed. The difficulty to detect the cytokines by using immunohistochemistry assay is also probably due to the characteristic of cytokines which is not fixed to the cell membrane.

The higher levels of sTNF, sTNF-R1 and sTNF$\mathrm{R} 2$ in RA than SpA could be due to the higher activity of TACE. TACE is responsible for the shedding of trans-membrane TNF, TNF-R1 and TNF-R2 into soluble form. ${ }^{4,6}$ As TACE cannot be detected in serum and SF, indirect investigation to detect TACE activity was performed. We first tested the SF levels of IL-6R. IL-6 and IL-6R can activate the signal transducer glycoprotein 130 (gp130) which contributes to an alternative pathway of joint erosions and increased bone resorption in RA. ${ }^{27,28}$ Furthermore, the trans-membrane form of IL-6R is also cleaved by TACE into a soluble form which 
can bind to IL-6. ${ }^{16}$ Our results showed that there was no difference of IL-6R level in synovial fluids from RA and SpA patients. TIMP-3 is known as a specific inhibitor of TACE activity. ${ }^{18}$ The level of TIMP-3 in synovial fluids from RA and SpA patients was also detected by using ELISA. It was found that the level of TIMP-3 in RA is significantly higher than in SpA. These two data indirectly showed that the lower levels of soluble form of TNF, TNF-R1 and TNF-R2 in SpA than in RA were probably not due to the lower TACE activity in SpA compared to RA. We are currently performing qPCR experiments to directly assess the expression of TACE as well as other proteinases in RA versus SpA synovium.

To see whether macrophages polarization has an influence on the disbalance of tmTNF and sTNF in SpA, we polarized macrophages in vitro into M1a (CAM), M2a and M2c (AAM) by IFNã, IL-4 and IL-10 stimulation, respectively. tmTNF expression by the polarized macrophages was assessed by flow cytometry. We confirmed that the expression of CD200R was upregulated by IL-4 stimulation. CD200R is an inhibitory macrophage receptor which is downregulated during classical macrophages activation. ${ }^{29,30} \mathrm{CD} 163$, another marker for alternative macrophages activation (AAM), was upregulated by IL-10 stimulation. Synovial infiltration with CD163+ AAM is associated with disease activity in peripheral SpA. ${ }^{25}$ Despite the fact that these data confirmed that the macrophages have been correctly polarized in our experiments, we were not able to detect the expression of tmTNF, even after additional LPS stimulation. Permeabilization of the cell membrane to detect the intracellular domain of transmembrane TNF did not improve the results. Our inability to detect tmTNF might be due to the fact that shedding of tmTNF into STNF by TACE happens within minutes. ${ }^{17}$

To confirm that the macrophages were able to produce TNF in our in vitro experiment, we performed ELISA for sTNF on the supernatant of these experiments. It is known that IFNã and IL-10 regulates macrophage functions in different ways. IFNã upregulates the production of many LPSinducible cytokines such as TNFá, IL-1â, IL-6 and IL-12 ${ }^{31,33}$ in monocytes while IL-10 downregulates the production of those cytokines..$^{34,35}$ This different role of IFNã and IL-10 was confirmed and extended by our results: LPS stimulation of IFNã polarized macrophages enhanced the production of TNF whereas IL-10 stimulation had the opposite effect. Although LPS and IL-4 costimulation suppresses the TNFá production by macrophages ${ }^{36}$, IL-4 polarization of macrophages enhanced the TNFá production induced by subsequent LPS stimulation.

\section{CONCLUSIONS}

In conclusion, the SF levels of sTNF, sTNF$\mathrm{R} 1$ and sTNF-R2 were significantly higher in RA than SpA. Interestingly, the sTNF-R2/sTNF-R1 was significantly higher in $\mathrm{SpA}$ than other groups suggesting that sTNF-R2 activity is relatively predominant in SpA pathology. This hypothesis is supported by the lower TNF-R1 mRNA levels in SpA synovial tissue. Taken together, this suggests that tmTNF/TNF-R2 signaling predominate over sTNF/TNF-R1 signaling in SpA synovitis as compared to RA synovitis.

Ongoing and future studies should address 3 main issues. Firstly, we are in the process of confirming these data by additional immunohistochemistry experiments. Secondly, the mechanism underlying this disbalance has not yet been elucidated. A differential expression or activation of TACE between SpA and RA is unlikely due to our sIL-6R and TIMP-3 results but this requires further confirmation. Alternatively, the differential expression of tmTNF and TNF-R by specific macrophage subsets is currently under investigation. Thirdly and finally, the most important issue is whether this disbalance has functional consequences for the pathology. Although this remains difficult to address mechanistically in translational human studies, there are some published evidences that this disbalance may affect crucial processes in the pathophysiology of arthritis. Our finding that the relative expression of TNF-R2/ TNF-R1 ratio which was lower in RA compared to $\mathrm{SpA}$ is in line with these data.

\section{ACKNOWLEDGEMENT}

We would like to thank Dominique L. Baeten and Cor Verweij for the great supervise and supports during my internship, Carmen A. Ambarus for your assistence during my work in the laboratory. We would like also to thank Nataliya Yaramenko for 
the cohort data and qPCR experiment and all people in Spondyloarthritis Groups of Clinical Immunology and Rheumatology Department of Amsterdam Medical Centre for supportive helps and positive input during my work.

\section{REFERENCES}

1. Carswell, E.A., et al. An endotoxin-induced serum factor that causes necrosis of tumors. Proc Natl Acad Sci U S A. 1975; 72(9): p. 3666-70.

2. Fiers, W. Tumor necrosis factor. Characterization at the molecular, cellular and in vivo level. FEBS Lett.1991; 285(2): p. 199-212.

3. Kriegler, M., et al. A novel form of TNF/ cachectin is a cell surface cytotoxic transmembrane protein: ramifications for the complex physiology of TNF. Cel. 1988; 53(1): p. 45-53.

4. Black, R.A., et al. A metalloproteinase disintegrin that releases tumour-necrosis factoralpha from cells. Nature.1997; 385(6618): p. 729-33.

5. Smith, C.A., T. Farrah, and R.G. Goodwin, The TNF receptor superfamily of cellular and viral proteins: activation, costimulation, and death. Cell. 1994; 76(6): p. 959-62.

6. Wang, J., et al. Histamine antagonizes tumor necrosis factor (TNF) signaling by stimulating TNF receptor shedding from the cell surface and Golgi storage pool. J Biol Chem. 2003; 278(24): p. 21751-60.

7. Grell, M., et al. The transmembrane form of tumor necrosis factor is the prime activating ligand of the $80 \mathrm{kDa}$ tumor necrosis factor receptor. Cell. 1995; 83(5): p. 793-802.

8. Gordon, S. and P.R. Taylor. Monocyte and macrophage heterogeneity. Nat Rev Immunol. 2005; 5(12): p. 953-64.

9. Haringman, J.J., et al. Synovial tissue macrophages: a sensitive biomarker for response to treatment in patients with rheumatoid arthritis. Ann Rheum Dis. 2005; 64(6): p. 834-8.

10.Kruithof, E., et al. Identification of synovial biomarkers of response to experimental treatment in early-phase clinical trials in spondylarthritis. Arthritis Rheum. 2006; 54(6): p. $1795-804$.

11.Campbell, I.K., L.J. Roberts, and I.P. Wicks. Molecular targets in immune-mediated diseases: the case of tumour necrosis factor and rheumatoid arthritis. Immunol Cell Biol. 2003; 81(5): p. 35466.

12.Milla, M.E., et al. Specific sequence elements are required for the expression of functional tumor necrosis factor-alpha-converting enzyme (TACE). J Biol Chem. 1999; 274(43): p. 3056370.

13.Moss, M.L., et al. Cloning of a disintegrin metalloproteinase that processes precursor tumour-necrosis factor-alpha. Nature. 1997; 385(6618): p. 733-6.

14.Peschon, J.J., et al. An essential role for ectodomain shedding in mammalian development. Science. 1998; 282(5392): p. 12814.

15.Black, R.A. Tumor necrosis factor-alpha converting enzyme. Int $\mathrm{J}$ Biochem Cell Biol. 2002; 34(1): p. 1-5.

16.Franchimont, N., et al. Interleukin-6 receptor shedding is enhanced by interleukin-1beta and tumor necrosis factor alpha and is partially mediated by tumor necrosis factor alphaconverting enzyme in osteoblast-like cells. Arthritis Rheum. 2005; 52(1): p. 84-93.

17.Fan, H. and R. Derynck. Ectodomain shedding of TGF-alpha and other transmembrane proteins is induced by receptor tyrosine kinase activation and MAP kinase signaling cascades. EMBO J. 1999; 18(24): p. 6962-72.

18. Amour, A., et al. TNF-alpha converting enzyme (TACE) is inhibited by TIMP-3. FEBS Lett. 1998; 435(1): p. 39-44.

19.Arend, W.P. and J.M. Dayer. Inhibition of the production and effects of interleukin- 1 and tumor necrosis factor alpha in rheumatoid arthritis. Arthritis Rheum. 1995; 38(2): p. 151-60.

20.Keller, C., A. Webb, and J. Davis. Cytokines in the seronegative spondyloarthropathies and their modification by TNF blockade: a brief report and literature review. Ann Rheum Dis. 2003; 62(12): p. 1128-32. 
21.Baeten, D., et al. Macrophages expressing the scavenger receptor CD163: a link between immune alterations of the gut and synovial inflammation in spondyloarthropathy. J Pathol. 2002; 196(3): p. 343-50.

22.Baeten, D., et al. Diagnostic classification of spondylarthropathy and rheumatoid arthritis by synovial histopathology: a prospective study in 154 consecutive patients. Arthritis Rheum. 2004; 50(9): p. 2931-41.

23.Mantovani, A., A. Sica, and M. Locati. Macrophage polarization comes of age. Immunity. 2005; 23(4): p. 344-6.

24. Vandooren, B., et al. Absence of a classically activated macrophage cytokine signature in peripheral spondylarthritis, including psoriatic arthritis. Arthritis Rheum. 2009; 60(4): p. 96675.

25.Baeten, D., et al. Infiltration of the synovial membrane with macrophage subsets and polymorphonuclear cells reflects global disease activity in spondyloarthropathy. Arthritis Res Ther. 2005; 7(2): p. R359-69.

26.Abu-Amer, Y., et al. Tumor necrosis factor receptors types 1 and 2 differentially regulate osteoclastogenesis. J Biol Chem. 2000; 275(35): p. 27307-10.

27.Naka, T., N. Nishimoto, and T. Kishimoto. The paradigm of IL-6: from basic science to medicine. Arthritis Res. 2002; 4 Suppl 3: p. S23342.

28.Wong, P.K., et al. The role of the interleukin-6 family of cytokines in inflammatory arthritis and bone turnover. Arthritis Rheum. 2003; 48(5): p. 1177-89.
29.Jenmalm, M.C., et al. Regulation of myeloid cell function through the CD200 receptor. J Immunol. 2006; 176(1): p. 191-9.

30. Wright, G.J., et al. Characterization of the CD200 receptor family in mice and humans and their interactions with CD200. J Immunol. 2003; 171(6): p. 3034-46.

31.Hayes, M.P., S.L. Freeman, and R.P. Donnelly. IFN-gamma priming of monocytes enhances LPS-induced TNF production by augmenting both transcription and MRNA stability. Cytokine. 1995; 7(5): p. 427-35.

32.Burchett, S.K., et al. Regulation of tumor necrosis factor/cachectin and IL-1 secretion in human mononuclear phagocytes. J Immunol. 1988; 140(10): p. 3473-81.

33.Hayes, M.P., J. Wang, and M.A. Norcross. Regulation of interleukin-12 expression in human monocytes: selective priming by interferon-gamma of lipopolysaccharideinducible p35 and p40 genes. Blood. 1995; 86(2): p. 646-50.

34.Bogdan, C., Y. Vodovotz, and C. Nathan. Macrophage deactivation by interleukin 10. J Exp Med. 1991; 174(6): p. 1549-55.

35.de Waal Malefyt, R., et al. Interleukin 10(IL-10) inhibits cytokine synthesis by human monocytes: an autoregulatory role of IL-10 produced by monocytes. J Exp Med. 1991; 174(5): p. 120920.

36.Hart, P.H., et al. Potential antiinflammatory effects of interleukin 4: suppression of human monocyte tumor necrosis factor alpha, interleukin 1, and prostaglandin E2. Proc Natl Acad Sci U S A. 1989; 86(10): p. 3803-7. 